\title{
RACSAM
}

Rev. R. Acad. Cien. Serie A. Mat.

VOL. 102 (1), 2008, pp. 101,125

Geometría y Topología / Geometry and Topology

Artículo panorámico / Survey

\section{Geometrization of three manifolds and Perelman's proof}

\author{
Joan Porti
}

\begin{abstract}
This is a survey about Thurston's geometrization conjecture of three manifolds and Perelman's proof with the Ricci flow. In particular we review the essential contribution of Hamilton as well as some results in topology relevants for the proof.
\end{abstract}

\section{Geometrización de variedades tridimensionales y la demostración de Perelman}

Resumen. Ésta es una exposición sobre la conjetura de geometrización de Thurston para variedades tridimensionales, así como de la demostración de Perelman mediante el flujo de Ricci. En particular se revisan la contribución esencial de Hamilton y algunos resultados de topología relevantes para la demostración.

\section{Introduction}

The aim of this paper is to give a brief description of Perelman's proof of Thurston's geometrization conjecture.

Theorem 1 (Perelman) Thurston's geometrization conjecture for three manifolds holds true.

A particular case is Poincaré conjecture, whose proof required almost a century, since it was raised in 1904 by Poincaré as a question [44].

During the first two thirds of XXth century, topological techniques specific for three manifolds where developed, obtaining some results that have been crucial for the final solution of geometrization. In particular, compact three manifolds where shown to be triangulable by Bing and Moise [3, 31], and admit unique smooth structures by Munkres [37], allowing the use of combinatorial and geometric techniques for topological conclusions.

Despite the previous results, during the 1960's and 1970's Poincaré conjecture seemed still out of reach, and there was not consensus whether it was true or false. The breakthrough came with W. P. Thurston, who proposed a geometric picture of three manifolds. His conjecture included Poincaré's as a particular case; and the evidences Thurston gave to support his own conjecture made people believe seriously in Poincaré's. More or less at the same time, R. H. Hamilton introduced the Ricci flow, proving Thurston's conjecture for manifolds equipped with a metric of nonnegative curvature. He also developed a program to prove geometrization conjecture, but the singularities where not well understood. In the electronic preprints of 2002 and 2003 [41 42 43], Perelman introduced new Riemannian techniques to understand the singularities

Presentado por José María Montesinos.

Recibido: 1 de septiembre de 2007. Aceptado: 5 de diciembre de 2007.

Palabras clave / Keywords: three manifolds, geometrization, Hamilton-Ricci flow

Mathematics Subject Classifications: 53C44, 57M50

(C) 2008 Real Academia de Ciencias, España. 
and gave a complete proof of geometrization. In particular he introduced the so called $\kappa$-non collapse results, that allow to continue and finish Hamilton's program. Those preprints are extremely concise, even sketchy many times, with almost no details but containing all the key points, so that it took few years to the community to accept the proof.

At the moment of writing, the proof is considered to be complete by many mathematicians. Besides Perelman's preprints [41 42] 43], the reader can find the notes [24] 33] 5]. In particular the notes of Kleiner and Lott [24] cover all aspects of the proof or give an appropriate reference, except for the collapse of the long term flow, that we discuss in Section 7.2 For this part we propose and approach in [1], which will be integrated in a book in preparation about Perelman's proof of geometrization.

A complete proof goes beyond the scope of this paper, the purpose is just to give an introduction to the conjecture and to sketch the results of Hamilton and Perelman that lead to the proof. In Chapter 2 we explain Thurston's geometrization conjecture, including few applications of geometrization and of Perelman's proof. Next chapters are devoted to Ricci flow, the work of Hamilton is described in Chapter 3 and part of 4 Perelman's work on singularities in the first preprint of Perelman [41] is very briefly described in Chapter 4 Chapter 5 is devoted to the flow with cutoff (a surgery process to deal with the singularities and continue the flow), which is the content of the first half of the second of Perelman's preprints [42]. Either the flow with cutoff becomes extinct in finite time or continues until infinity. In Chapter 6 we explain why it becomes extinct for manifolds with finite fundamental group and gives a "shortcut" for the proof of Poincaré. This is the content of the third preprint of Perelman [41], but here we follow [10]. Finally in Chapter 7 we explain the long time behaviour, following the second half of [42], and we explain the alternative approach to collapse that we propose in [1].

\section{Thurston's Geometrization Conjecture}

In this chapter we explain the geometrization conjecture. In dimension two, every closed surface admits a metric of constant curvature; this result can be seen as a uniformization theorem. In dimension three, there are topological obstructions for having such a metric, or even for having a metric locally homogeneous (the so called geometric manifolds). Thurston's geometrization conjecture asserts that every three manifold has a canonical decomposition in geometric pieces [52]. Before Thurston's, we explain Poincaré's.

\subsection{Poincaré Conjecture}

This conjecture is not only a particular case of the geometrization conjecture, but it has been a strong motivation for research in the field along the XXth century.

The conjecture is the positive answer to the question that Poincaré raised in 1904 at the end of the paper "cinquième complement à l' analysis situs" [44]. It seems that Poincaré never conjectured any answer to his question, he just wrote at the end of the paper: "mais cette question nous entrainerait trop loin". In modern language, it can be stated as:

Poincaré's question Is the sphere the only three dimensonal manifold with trivial fundamental group?

For an overview of the work on this conjecture before 1980 the reader may read [32]. Here we will start with the work of Thurston at end of the 1970's. However the topological preliminaries go back to Kneser in 1928.

\subsection{Topological decomposition}

As mentioned above, there are topological obstructions for a three manifold to admit a locally homogeneous metric. Here we explain, the so called canonical topological decomposition, which has two steps.

Connected sum is the first step in the topological decomposition. Recall that, in any dimension, the connected sum of two manifolds consists in removing an open ball of each one and to glue them along the boundary spheres. 
For every manifold, the connected sum with the sphere of the same dimension does not change the manifold, because a sphere is the result of gluing two balls along the boundary. We say that a manifold is prime if it is not the connected sum of two manifolds different from the sphere.

The first step in the canonical decomposition was proved by Kneser [25], for the existence, and Milnor, for the uniqueness [30].

Theorem 2 (Kneser 1928, Milnor 1962) Every closed, oriented three-manifold $M^{3}$ can be decomposed as a connected sum

$$
M^{3}=M_{1}^{3} \# \cdots \# M_{k}^{3}
$$

so that each $M_{i}^{3}$ is prime. In addition, the manifolds $M_{i}^{3}$ are unique up to permutation and homeomorphism.

Instead of prime, for technical reasons it is easier to work with irreducible manifolds.

Definition 1 A 3-manifold $M^{3}$ is called irreducible if every embedded 2-sphere in $M^{3}$ is the boundary of an embedded 3-ball in $M^{3}$.

The following remark is well know, and allows to work with irreducible manifolds.

Remark 1 Let $M^{3}$ be a closed orientable 3-manifold. Then $M^{3}$ is prime if and only if either $M^{3}$ is irreducible or $M^{3} \cong S^{2} \times S^{1}$.

Later in the 1930's, H. Seifert introduced a family of manifolds, that he called fibered, nowadays known as Seifert manifolds [48].

A Seifert fibration is a partition by circles (locally a product) except for a finite number of singular fibres, that have the following local model. We consider a cylinder, the product $D^{2} \times[0,1]$ of a disc with an interval, and we glue $D^{2} \times\{0\}$ with $D^{2} \times\{1\}$ by a rotation of finite order (cf. Figure 1a). The fibration by horizontal intervals induces a partition by circles of the solid torus (cf. Figure lb), that is a fibration except for the singular fibre $\{0\} \times S^{1}$, which is shorter.

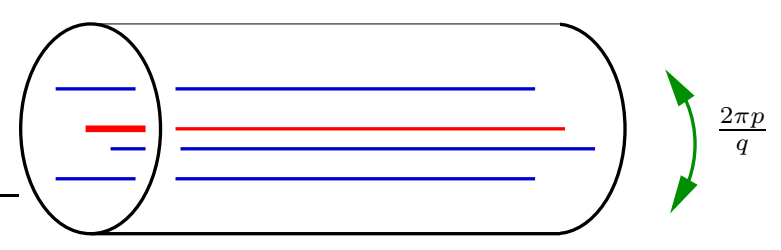

(a)

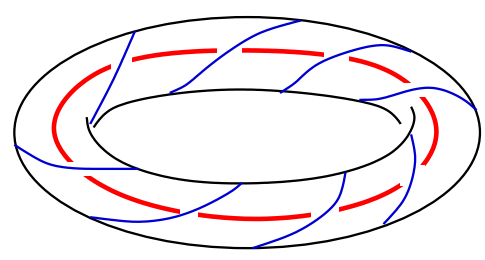

(b)

Figure 1: Model for the Seifert fibration. We glue the left and right side of the cylinder $[0,1] \times D^{2}$ in (a) by a rotation of angle $2 \pi \frac{p}{q}$, with $\frac{p}{q} \in \mathbb{Q}$

Seifert fibrations are described by the the space of fibers, the coefficients $(p, q)$, and a rational Euler class. Seifert classified topologically the compact three manifolds admiting a Seifert fibration [48], in particular proving Poincaré conjecture for this family of manifolds.

During the 1970's, W. Jaco and P. Shalen from one side, and K. Johannson independently, they found a canonical decomposition along tori, currently know as JSJ-decomposition.

Theorem 3 (Jaco-Shalen and Johansonn 1979) Let $M^{3}$ be an irreducible, closed and orientable three manifold $M^{3}$. There exists a family of incompressible tori $T_{1}^{2}, \ldots, T_{k}^{2}$ that cut $M^{3}$ into pieces that are either Seifert fibered or simple. The family of tori is canonical if minimal.

The torus of the family are assumed to be embedded and disjoint. "Canonical" means unique up to isotopy. 
Definition 2 A tori $T^{2} \subset M^{3}$ is said to be incompressible if $\pi_{1}\left(T^{2}\right) \hookrightarrow \pi_{1}\left(M^{3}\right)$ is injective.

An irreducible manifold $M^{3}$ is called simple if it is not Seifert fibered and every subgroup $\mathbf{Z} \oplus \mathbf{Z}<$ $\pi_{1} M^{3}$ comes from a component of the boundary $\partial M^{3}$.

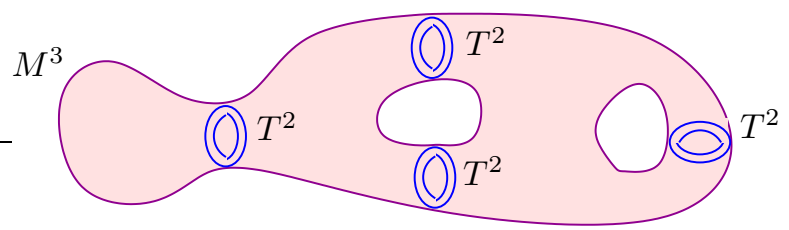

Figure 2: JSJ decomposition along tori

Because of geometrization conjecture, this theorem is known as the JSJ splitting, but it is also known as the characteristic submanifold theorem. In fact, Jaco, Shalen, and Johansonn found a maximal canonical Seifert submanifold, and they called the remaining pieces simple. We will see however that in many aspects, they have a more complicated topology.

\subsection{Geometrization}

According to the JSJ decomposition (Thm. 3), the manifolds remaining to be understood after that were the simple ones. Thuston's conjectured that simple manifolds are hyperbolic [52].

Definition 3 A manifold is said to be hyperbolic if its interior has a complete metric of constant curvature -1 .

It is important to notice that manifolds with finite fundamental group cannot be hyperbolic. Hence the conjecture claims that irreducible manifolds with finite fundamental group are Seifert fibered. It is clearer to state two conjectures, according to whether the fundamental group of the manifold is finite or infinite.

Hyperbolization Conjecture Let $M^{3}$ be a prime, closed three-manifold, with $\pi_{1}\left(M^{3}\right)$ infinite and such that every subgroup $\mathbf{Z} \oplus \mathbf{Z}<\pi_{1}\left(M^{3}\right)$ comes from $\partial M^{3}$. Then $M^{3}$ is hyperbolic.

Definition 4 A 3-manifold is said to be elliptic if it admits a metric of constant curvature +1.

If $M^{3}$ is a closed three manifold and $\pi_{1} M^{3}$ is finite, then equipping $M^{3}$ with an elliptic metric is equivalent to admit a Seifert fibration, using the fact both families elliptic manifolds and Seifert fibered ones are classified, [47].

Elliptization Conjecture Let $M^{3}$ be a prime, closed three-manifold, with $\pi_{1}\left(M^{3}\right)$ finite. Then $M^{3}$ is elliptic.

This conjecture subsumes Poincaré, as it includes the case with trivial fundamental group. Both conjectures can be joint in a single one:

Thurston's Geometrization Conjecture (first version) Every closed orientable three manifold decomposes canonically into pieces whose interior is either Seifert fibered or hyperbolic

Of course the canonical decomposition is the one due to Kneser and JSJ.

This is not still the usual version of the conjecture. We need a couple of definitions.

Definition 5 A metric is said to be homogeneous when the isometry group acts transitively, i.e. any two points are related by an isometry of the manifold. When such an isometry is only local, defined only in a neighborhood of the points, then the metric is said to be locally homogeneous. 
The conjecture it is better know as follows:

Thurston's Geometrization Conjecture (second version) Every closed orientable three manifold decomposes canonically into pieces whose interior has a locally homogeneous complete metric.

The equivalence between both formulations of the conjecture comes from:

Proposition 1 A 3-manifold admits a locally homogeneous metric if and only if it is either hyperbolic, Seifert fibered, or a torus bundle over $S^{1}$.

This proposition uses the classification of homogeneous metrics on simply connected three-manifolds done by L. Bianchi [2] in 1897, with cosmological motivations.

\subsection{Examples}

Example 1 Consider the unit sphere $S^{3} \subset \mathbb{R}^{4}$. It has an homogeneous metric, as the isometry group is the orthogonal group $O(4)$.

To illustrate Proposition 1 we see the sphere as a fibered space. In fact we see $S^{3}$ as the unit sphere in $\mathbb{C}^{2}$,

$$
S^{3}=\left\{\left(z_{1}, z_{2}\right) \in \mathbb{C}^{2} \mid z_{1} \bar{z}_{1}+z_{2} \bar{z}_{2}=1\right\} .
$$

The fibres of the Hopf fibration are the orbits of the action of

$$
S^{1}=\{\omega \in \mathbb{C} \mid \omega \bar{\omega}=1\}
$$

by left multiplication: i.e. the fibres are the circles

$$
\left\{\left(e^{i \theta} z_{1}, e^{i \theta} z_{2}\right) \mid e^{i \theta} \in S^{1}\right\} .
$$

The space of fibres is therefore the projective line $\mathbb{C} \mathbf{P}^{1}$.

Example 2 We consider next Lens spaces $L(p, q)$, where $p, q \in \mathbb{Z}$ denote coprime integer numbers, and $p \geq 2$. Following the notation of previous example, $L(p, q)$ is the quotient of $S^{3}$ by the cyclic action of order $p$ generated by the transformation

$$
\left(z_{1}, z_{2}\right) \mapsto\left(e^{2 \pi i / p} z_{1}, e^{2 \pi i q / p} z_{2}\right)
$$

This action preserves the metric of $S^{3}$, hence it induces a metric on constant curvature in $L(p, q)$. In addition this action conmutes with the multiplication of the Hopf fibration, and permutes orbits, except for two orbits that are invariant: $\left\{\left(0, z_{2}\right) \mid z_{2} \in S^{1}\right\}$ and $\left\{\left(z_{1}, 0\right) \mid z_{1} \in S^{1}\right\}$. Therefore $L(p, q)$ admits a Seifert fibration with two singular orbits of order $p$.

It can be shown that all elliptic manifolds, i.e. quotients of $S^{3}$ by a finite subgroup of $O(4)$ admit a Seifert fibration induced by the Hopf fibration [47].

Example 3 Consider $F_{g} \times S^{1}$, the product of a closed surface of genus $g \geq 1$ with the circle. Since the surface $F_{g}$ admits a metric of constant curvature, the product metric $F_{g} \times S^{1}$ is locally homogeneous. It is obviously Seifert fibered.

Example 4 Let us consider the Heisenberg group:

$$
\text { Heis }=\left\{\left(\begin{array}{lll}
1 & x & z \\
0 & 1 & y \\
0 & 0 & 1
\end{array}\right) \mid x, y, z \in \mathbb{R}\right\}
$$

equipped with a left invariant metric, and the subgroup

$$
\Gamma=\left\{\left(\begin{array}{lll}
1 & x & z \\
0 & 1 & y \\
0 & 0 & 1
\end{array}\right) \mid x, y, z \in \mathbb{Z}\right\} .
$$


The quotient $\Gamma \backslash$ Heis inherits a locally homogeneous metric. In addition, the projection to the coordinates $(x, y)$ gives a fibration by circles over the torus

$$
\begin{aligned}
& S^{1} \rightarrow \text { Heis } / \Gamma \quad \rightarrow \quad T^{2}=\mathbb{R}^{2} / \mathbb{Z}^{2} \\
& (x, y, z) \mapsto(x, y)
\end{aligned}
$$

Example 5 The figure eight knot (Fig. 3) is the simplest example of hyperbolic knot, i.e. a knot in $S^{3}$ whose complement is a complete hyperbolic manifold of finite volume [45, [51].

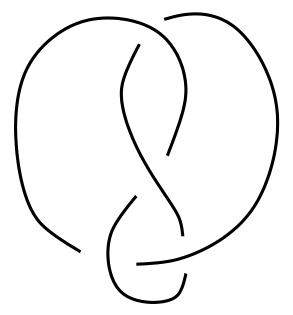

Figure 3: The figure eight knot

We always represent knots in the three space, understanding that they lie in its one point compactification $\mathbb{R}^{3} \cup\{\infty\}=S^{3}$.

Example 6 The next example is also the exterior of a knot in $S^{3}$, and has a nontrivial JSJ decomposition. The knot in Figure 4 is called a satellite of the figure eight knot, because it lies in the tubular neighborhood of the figure eight knot. The boundary of this tubular neighborhood is a torus $T^{2}$, that gives a JSJ decomposition. Outside $T^{2}$, we have the exterior of the figure eight knot, which is hyperbolic. Inside $T^{2}$, we have a Seifert manifold, since we can assume that this piece is the result of removing a solid torus from a model.
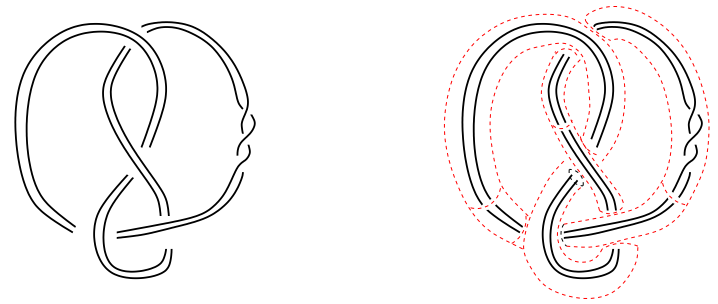

Figure 4: A satellite of the figure eight knot. On the right hand picture, the torus $T^{2}$ of the JSJ decomposition is represented

Example 7 Let $F_{g}^{2}$ be a surface of genus $g \geq 2$. Thurston classified in [56] the automorphisms $\phi: F^{2} \rightarrow$ $F^{2}$ in three families: (a) periodic or trivial, (b) reducible (i.e. having a non-trivial closed invariant curve in $F^{2}$ ) or (c) pseudo-anosov. This classification has a correspondence with the decomposition in geometric pieces of the mapping torus $M_{\phi}^{3}=F^{2} \times[0,1] /(x, 1) \sim(\phi(x), 0): M_{\phi}^{3}$ is Seifert fibered in case $(a)$, it contains an essential torus in case (b) and it is hyperbolic in case (c). The later is a particular case of Thurston's hyperbolization theorem [39, 54].

\subsection{Some partial results about geometrization known before Perelman}

Here we list some results that may be viewed as a partial positive answer to geometrization, though they originally could have been formulated with a different purpose. This list is far from beeing complete, we 
just quote few of them. In this section we also omit the results of Hamilton [17, 18], that will be explained in Theorem 16 and Remark 2

We start with a result of Waldhausen.

Definition 6 A 3-manifold is called a graph manifold if it is a union of Seifert manifolds along the boundary, consisting of tori.

Notice that those gluing tori do not need to be the ones of the JSJ decomposition, as the manifold is not assumed to be irreducible, and the tori are not supposed to be incompressible. Waldhausen proved in [58] a theorem that can be viewed as a precedent of the JSJ decomposition, and that covers one case of geometrization. The modern version is the following:

Theorem 4 (Waldhausen 1967) A closed orientable graph 3-manifold is the connected sum of manifolds whose JSJ decomposition contains only Seifert manifolds.

The next result is Thurston's geometrization for sufficiently large manifolds, that was one of the main evidences to support his conjecture.

Definition 7 An orientable 3-manifold is called sufficiently large if it contains an properly embedded incompressible surface (i.e. $\pi_{1}$-injective or a disc not parallel to the boundary)

A surface $F^{2} \subset M^{3}$ is called properly embedded if it is embedded and $\partial F=F \cap \partial M^{3}$.

Haken [16] proved that sufficiently large manifolds have a hierarchy: one can cut them successively along incompressible surfaces to end up with three-balls, and this is why they are often called Haken manifolds. Using those hierarchies Thurston proved:

Theorem 5 (Thurston 1981) A sufficiently large three manifold satisfies the geometrization conjecture.

For the complete proof of this theorem, we refer to Thurston's papers and preprints [53 [54 [55] as well as [23, 26, 34, 39 40]. The proof involves many different techniques and has stimulated research in Kleinian groups, representation spaces, $\mathbb{R}$-trees and surface automorphisms. One of the most notable results about sufficiently large manifolds was proved by Waldhausen [59]:

Theorem 6 (Waldhausen 1968) Any homotopy equivalence relative to the boundary between suffciently large three manifolds can be deformed to a homeomorphism.

The following theorem is the result of work by Casson-Jungreis, Gabai, Mess, Scott and Tukia, [47, 13. 57, 6, 29] and it involves convergence groups.

Theorem 7 Let $M^{3}$ be a compact irreducible 3-manifold. If $\pi_{1}\left(M^{3}\right)$ has an infinite cyclic normal subgroup, the $M^{3}$ is Seifert fibered.

Among other results of Thurston supporting his conjecture, we quote the orbifold theorem. An orbifold is like a manifold but modeled in finite quotients of $\mathbb{R}^{n}$ instead of just $\mathbb{R}^{n}$. Thus an orbifold has a branching locus, corresponding to the points branching points of the model.

For a proof of the following theorem, the reader can check [4] and references therein.

Theorem 8 (Thurston) Any compact irreducible and orientable three orbifold with non-empty branching locus satisfies the geometrization conjecture.

In the three dimensional setting, Cheeger-Gromov theory for collapses can also be seen as a geometrization result. Here $\operatorname{inj}(x)$ denotes the injectivity radius of a point in a Riemannian manifold, and $\sec (x)$ its sectional curvature. Let us state the tridimensional version of Cheeger and Gromov's theorem [8].

Theorem 9 (Cheeger-Gromov) There exists a universal constant $\varepsilon>0$ such that, for every three dimensional Riemannian manifold $M^{3}$, the subset

$$
\left\{x \in M^{3} \mid \operatorname{inj}(x) \leq \varepsilon \text { and }|\sec (x)| \leq 1\right\}
$$

is a graph manifold.

In addition, for every $\varepsilon>0$, any graph manifold admits Riemannian metric with $|\sec (x)| \leq 1$ and $\operatorname{inj}(x) \leq \varepsilon$ at every point $x$. 


\subsection{Consequences of Geometrization}

Some consequences of geometrization in three dimensional topology are listed in this section. The list could be much enlarged, specially if entering in technical questions of three dimensional topology.

Definition 8 A Whitehead manifold is a contractible 3 -manifold which is not homeomorphic to $\mathbb{R}^{3}$.

Such a manifold must be necessarily open, otherwise it would be a counterexample to Poincaré conjecture. The first example was constructed by J. H. C. Whitehead himself in 1935 [60]. Previously, Whitehead had published a wrong proof of Poincaré conjecture, which included the generalization of the conjecture for open manifolds. He found himself the mistake and the counterexample to the wrong statement, the Whitehead manifold.

Theorem 10 No Whitehead manifold is the universal covering of a compact three manifold.

This is equivalent to say that the universal covering of a compact aspherical 3-manifold must be $\mathbb{R}^{3}$. The theorem is well know if the manifold is geometric, because $\mathbb{R}^{3}$ is the only simply connected manifold with an homogeneous Riemannian metric. For an aspherical three-manifold $M^{3}$ in general, we first notice that it must be irreducible, as $\pi_{2}\left(M^{3}\right)=0$. Hence if it is not geometric, then $M^{3}$ has a nontrivial JSJ decomposition. In particular $M^{3}$ is sufficiently large and in this case this has been proved by Waldhausen [59].

The folowing is known as Borel conjecture, which remains widely unknown in higher dimension.

Theorem 11 (Borel conjecture in dimension three) If two aspherical compact three manifolds are homotopically equivalent, then they are homeomorphic.

If the manifolds are geometric, this follows from Mostow rigidity [35] in the hyperbolic case, and from Seifert's work [48] in the Seifert fibered case. Otherwise, as in the proof of Theorem 10] the manifolds are irreducible, and they have a nontrivial JSJ decomposition. Thus they are sufficiently large and one can apply Waldhausen's theorem (Thm. 6.

Of course asphericity is required, as there exist lens spaces which are homotopically equivalent but not homeomorphic. The equivalent formulation is the following one:

Theorem 12 Compact aspherical three manifolds are classified by its fundamental group.

Another important consequence is the uniformization of actions on the three sphere. Together with the orbifold theorem, geometrization implies:

Theorem 13 Every finite subgroup of orientation preserving diffeomorphisms of $S^{3}$ is conjugate to a finite subgroup of $S O(4)$.

\subsection{Consequences of Perelman's proof}

Before explaining Perelman's proof, we give some of its consequences. We start with the following result, whose version up to homotopy was proven by Gromov-Lawson [15] and Schoen-Yau [46].

Corollary 1 If a compact three manifold admits a metric of non-negative scalar curvature then it is either flat or a connected sum of elliptic manifolds and $S^{2} \times S^{1}$ or its quotients.

Here is another consequence of the approach follwed by [1]. For a manifold $M^{3}$ one can define a topological invariant as follows: given a compact Riemannian manifold $\left(M^{3}, g\right)$, let $R_{\min }\left(M^{3}, g\right)$ denote the minimum of the scalar curvature, and

$$
\hat{R}\left(M^{3}, g\right)=R_{\min }\left(M^{3}, g\right) \operatorname{Vol}\left(M^{3}, g\right)^{2 / 3}
$$


the normalization of the minimum of the scalar curvature. This normalization by the $2 / 3$-th power of the volume is invariant by homotety. In adition, when it is negative, it is uniformly growing by the Ricci flow. We also denote

$$
\hat{R}\left(M^{3}\right)=\sup _{g} \hat{R}\left(M^{3}, g\right) \text {. }
$$

According to [24 (93.6)] $\hat{R}\left(M^{3}\right)$ equals the Yamabe invariant when it is non-positive.

On the other hand, for a three manifold $M$ one can define a topological invariant:

$$
V_{0}(M)=\inf \{\operatorname{Vol}(M \backslash L) \mid L \text { is a link or empty, with } M \backslash L \text { hyperbolic }\}
$$

By a theorem of Myers [38], such a link always exists, and by Mostow Rigidity, it is a topological invariant. Using the Jørgensen-Thurston structure theorem for the volume manifolds [52], this infimum is always reached by a positive minimum.

Theorem 14 ([1]) Let $M^{3}$ be a compact irreducible three manifold with infinite fundamental group. Then

$$
\hat{R}(M) \leq-6 V_{0}(M)^{2 / 3}
$$

with equality if and only if $M$ is hyperbolic.

\section{The Ricci flow}

Before defining the flow, we recall the different curvatures. We start with the tensor $R(\cdot, \cdot) \cdot$. For any three fields $X, Y$ and $Z$ in a manifold, $R(X, Y) Z$ is the field:

$$
R(X, Y) Z=\nabla_{X, Y}^{2} Z-\nabla_{Y, X}^{2} Z=\nabla_{X} \nabla_{Y} Z-\nabla_{Y} \nabla_{X} Z-\nabla_{[X, Y]} Z
$$

When we take the trace we get the Ricci curvature:

$$
\operatorname{Ric}(X, Y)=\operatorname{Trace}(Z \mapsto R(X, Z) Y)
$$

the Ricci curvature is a symmetric tensor twice equivariant, hence of the same order as the metric tensor $g(\cdot, \cdot)$, which is also symmetric.

When we write Ric $\geq 0$ we mean that Ric is a positive semidefinite bilinear form, and positive definite when Ric $>0$.

Scalar curvature is the trace of the Ricci tensor, and it is usually denoted by $R$. It is not a tensor but a function, hence much easier to work with.

Sectional curvature is defined for each plane in the tangent space. Given two vectors $u$ and $v$, the sectional curvature of the plane they $\operatorname{span}\langle u, v\rangle$ is

$$
\sec (\langle u, v\rangle)=g(R(u, v) v, u)
$$

where $g$ denotes the metric tensor.

In dimension three, both the sectional curvatures and the Ricci curvature determine the whole curvature tensor. This is not true in higher dimension for Ricci curvature.

\subsection{The flow}

It was introduced by Hamilton in 1982, when he geometrized 3-manifolds with non-negative Ricci curvature [17]. The flow is defined by the equation

$$
\frac{\partial g}{\partial t}=-2 \text { Ric }
$$


where $g$ denotes a Riemannian metric and Ric its corresponding Ricci tensor. This is a differential equation in the space of symmetric 2 -tensors on the manifold. In coordinates $\left(x^{1}, \ldots, x^{n}\right)$, it is written as

$$
\frac{\partial g_{i j}}{\partial t}=-2 R_{i j}
$$

where $g_{i j}=g\left(\frac{\partial}{\partial x_{i}}, \frac{\partial}{\partial x_{j}}\right)$ and $R_{i j}=\operatorname{Ric}\left(\frac{\partial}{\partial x_{i}}, \frac{\partial}{\partial x_{j}}\right)$.

Theorem 15 (Short time existence [17, 12]) If $M$ is a compact manifold with a Riemannian metric $g_{0}$, then the equation has a unique solution for time $t \in[0, T)$ for some $T>0$.

To understand why this equation can be useful for the geometrization conjecture, note that in harmonic coordinates (i.e. $\Delta\left(x^{i}\right)=0$ ) the equation is

$$
\frac{\partial g_{i j}}{\partial t}=\Delta\left(g_{i j}\right)+Q_{i j}\left(g^{-1}, \frac{\partial g}{\partial x}\right)
$$

where $\Delta\left(g_{i j}\right)$ is the laplacian of the scalar function $g_{i j}$ and $Q_{i j}$ is a quadratic expression. This is a diffusionreaction equation, and the heuristics of Hamilton's program is the following: "Either $g(t)$ converges to a locally homogeneous metric or creates singularities corresponding to the canonical decomposition".

\subsection{Examples}

We start with the simplest example: assume that the initial metric $g_{0}$ has constant curvature $K$. Then, knowing the existence and uniqueness of solutions, we will restrict to homotetic metrics $g_{t}=f(t) g_{0}$ and find a solution among those. Since the Ricci tensor is invariant by homoteties,

$$
\operatorname{Ric}_{g_{t}}=\operatorname{Ric}_{g_{0}}=(n-1) K g_{0},
$$

where $n$ is the dimension, the equation becomes $f^{\prime}=-2(n-1) K$, hence the solution is

$$
g_{t}=(1-2 K(n-1) t) g_{0} .
$$

The behaviour depends on the sign of the curvature:

- For $K=0$, the solution is stable.

- For $K<0$ the solution expands for infinite time.

- For $K>0$ it collapses and shrinks to a point in finite time $T=\frac{1}{2 K(n-1)}$.

A more general family of solutions is given by solitons.

Definition 9 A solution $g_{t}$ to the flow is a soliton if $g_{t}=\lambda_{t} \phi_{t}^{*} g_{0}$, where $\phi_{t}$ is a diffeomorphism and $\lambda_{t}$ is a constant, both depending on $t$.

In the particular case where $\phi_{t}$ is the uniparametric group associated to a gradient vector field, we say that $g_{t}$ is a gradient soliton. Being a gradient soliton is equivalent to the existence of a function $f$ satisfying:

$$
\operatorname{Ric}+\operatorname{Hess}(f)+c g=0 .
$$

This equation is obtained by differentiating $g_{t}=\lambda_{t} \phi_{t}^{*} g_{0}$ at time 0 , and assuming that $\phi_{t}$ is the group of diffeomorphism obtained by integrating the field $\nabla f$. Here $c=\lambda_{0}^{\prime} / 2$, hence $c=0$ corresponds to a stationary soliton $(\lambda \equiv 1)$. 


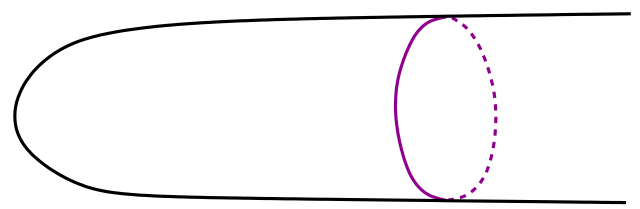

Figure 5: The cigar.

Example 8 The cigar soliton is the metric $g=\frac{d x^{2}+d y^{2}}{1+x^{2}+y^{2}}$ in $\mathbb{R}^{2}$ (Fig. [5). In polar coordinates centered at $(x, y)=(0,0)$, the metric of the cigar soliton is

$$
g=d r^{2}+\tanh ^{2} r d \theta .
$$

Notice that it is asymptotically a cylinder $(\tanh r \rightarrow 1$ when $r \rightarrow \infty)$. The sectional curvature is $\frac{2}{\cosh ^{2} r}$, hence $\operatorname{Ric}=\frac{2}{\cosh ^{2} r}$ g and $f=-2 \log \cosh r$ satisfies the gradient soliton equation $\operatorname{Ric}+\operatorname{Hess}(f)=0$.

Example 9 The cylinder $S^{2} \times \mathbb{R}$ with the standard metric is a contracting soliton, and the metric collapses in the $S^{2}$ direction in finite time (Fig. 6.

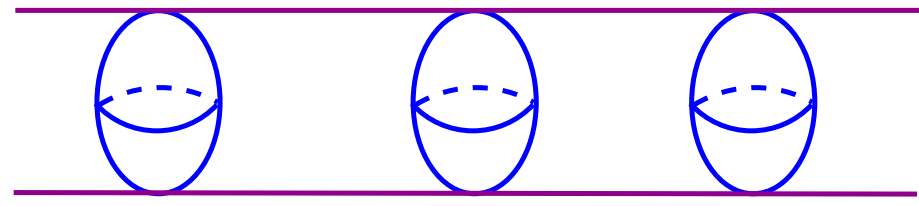

Figure 6: The cylinder.

The previous examples play a different role in Hamilton's program for the proof of the geometrization conjecture, in particular for the analysis of the singularities. The cylinder and the cigar times $\mathbb{R}$ are both examples of solutions to the Ricci flow which have nonnegative curvature, are complete and ancient (defined in at least a time interval $(-\infty, 0])$. Hamilton showed those kind of solutions appear as limits of singularities of the Rici flow on three dimensional manifolds, after rescaling (metric and time) and blowing up. Cylinders are convenient limits, as this should correspond to a connected sum decomposition on the manifold. To avoid the cigar times $\mathbb{R}$, Perelman introduces the notion of $\kappa$-non-collapse (Theorem 19 .

\subsection{Evolution of curvature}

The evolution of curvature is central in the study of the Ricci flow. We start with scalar curvature for simplicity

Lemma 1 The evolution of scalar curvature $R=\operatorname{trace}(\mathrm{Ric})=R_{i j} g^{i j}$ during the Ricci flow is:

$$
\frac{\partial R}{\partial t}=\Delta R+|\operatorname{Ric}|^{2}
$$

The weak and strong maximum principles applied to this equation yield:

Corollary 2 On a compact manifold $M, \min _{M} R$ is non-decreasing with time. 
Corollary 3 On a compact manifold $M$, if at time $t=0, R \geq 0$ everywere and $R>0$ for some $x \in M$, then $R>0$ everywhere when $t>0$.

Equations for the Ricci and the whole curvature tensor itself can be given. In the three dimensional case, the curvature operator (acting on 2-forms) diagonalizes:

$$
\left(\begin{array}{ccc}
\alpha_{1} & 0 & 0 \\
0 & \alpha_{2} & 0 \\
0 & 0 & \alpha_{3}
\end{array}\right)
$$

so that the $\alpha_{i}$ are functions on $M$. Then the $\alpha_{i} / 2$ are sectional curvatures,

$$
\operatorname{Ric}=\left(\begin{array}{ccc}
\frac{\alpha_{2}+\alpha_{3}}{2} & 0 & 0 \\
0 & \frac{\alpha_{1}+\alpha_{2}}{2} & 0 \\
0 & 0 & \frac{\alpha_{1}+\alpha_{2}}{2}
\end{array}\right)
$$

and $R=\alpha_{1}+\alpha_{2}+\alpha_{3}$.

The evolution equations for the $\alpha_{i}$ are

$$
\left\{\begin{array}{l}
\alpha_{1}^{\prime}=\Delta \alpha_{1}+\alpha_{1}^{2}+\alpha_{2} \alpha_{3} \\
\alpha_{2}^{\prime}=\Delta \alpha_{2}+\alpha_{2}^{2}+\alpha_{3} \alpha_{1} \\
\alpha_{3}^{\prime}=\Delta \alpha_{3}+\alpha_{3}^{2}+\alpha_{1} \alpha_{2}
\end{array}\right.
$$

To those kind of equations one applies the weak maximum principle for tensors, developed by Hamilton. One deduces for instance:

Proposition 2 In dimension three, Ric $\geq 0$, Ric $>0, \sec \geq 0$ and $\sec >0$ are conditions invariant under the Ricci flow.

Recal that Ric is a symetric bilinear form, and the inequalities in the statement of the proposition mean that it is positive semidefinite or definite, respectively

Maximum principles where also used by Hamilton to control the $\alpha_{i}$ in the following theorem.

Theorem 16 (Hamilton 1982 [17|) If a compact three manifold $M^{3}$ admits a metric with Ric $>0$, then the Ricciflow, after rescaling, converges to a metric with constant positive sectional curvature. In particular $M^{3}$ is elliptic.

Hamilton also developed a strong maximum principle for tensors [18], and used it to show that if Ric $\geq 0$, then one of the three possibilities happen:

1. The metric is flat.

2. Ric $>0$ at $t>0$, hence $M$ is elliptic.

3. The metric is locally a product $g=g_{1} \oplus d x^{3}$. In this case the manifold is diffeomorphic to $S^{2} \times S^{1}$ or it is a quotient $\mathbb{R} \mathbf{P}^{3} \# \mathbb{R} \mathbf{P}^{3}$.

Hence we conclude:

Remark 2 Hamilton had proved in 1984 [18] that a closed manifold with a Riemanian metric with Ric $\geq 0$ satisfies Thuston's geoemtrization conjecture. 


\subsection{Evolution of volume}

The evolution of curvature helps to study the behaviour of the volume. We start by giving the evolution equation for the volume.

Lemma 2 The volume evolves by the Ricci flow satisfying

$$
\frac{d}{d t} \operatorname{Vol}(M, g(t))=-\int_{M} R
$$

Corollary 4 If $g(t)$ evolves by the Ricci flow, then

$$
\operatorname{Vol}(M, g(t))(t+1 / 4)^{-3 / 2}
$$

is not increasing in time, provided that for the initial metric at time 0 we have

$$
R_{\min }(0)=\min _{(M, g(0))} R \geq-6 .
$$

Proof. Recall from Lemma 1 that $R$ evolves by:

$$
\frac{d}{d t} R=\Delta R+2|\mathrm{Ric}|^{2}
$$

since $R$ is the trace of Ric, decomposing Ric as the addition of a traceless part and a diagonal matrix, we have $\mid$ Ric $\left.\right|^{2} \geq \frac{1}{3} R^{2}$. Hence

$$
\frac{d}{d t} R \geq \Delta R+\frac{2}{3} R^{2}
$$

By the maximum principle, the minimum of the scalar curvature in $M$ at time $t, R_{\min }(t)$, satisfies:

$$
\frac{d}{d t} R_{\min } \geq \frac{2}{3} R_{\min }^{2}
$$

Using the normalization $R_{\min }(0) \geq-6$ for the initial metric, hence we get:

$$
R_{\min }(1) \geq-\frac{2}{3} \frac{1}{1+1 / 4}
$$

In addition, since the volume evolves by Lemma 2 it follows that:

$$
\frac{d}{d t} \operatorname{Vol}(M, g(t)) \leq-R_{\min }(t) \operatorname{Vol}(M, g(t)) .
$$

Combining those equations, we get that $\operatorname{Vol}(M, g(t))(t+1 / 4)^{3 / 2}$ is non increasing.

\subsection{Hamilton Ivey pinching of curvature}

Hamilton-Ivey estimates [19, 21] are another example of a clever application of maximum principles for tensors in dimension three. Let $\phi:[-1,+\infty) \rightarrow[1,+\infty)$ be the inverse map of $x \mapsto \log x-x$.

Theorem 17 (Hamilton-Ivey pinching) The inequalities

$$
R \geq-1 \quad \text { and } \quad \alpha_{1}, \alpha_{2}, \alpha_{3} \geq-\phi(R)
$$

are invariant under the Ricci flow. 
We remark that, given any metric, the inequalities of the theorem are satisfied after homotety. Hence from now on we shall assume that the flow starts with a normalized initial metric, i.e. satisfying those conditions at time 0 .

Since $R=\alpha_{1}+\alpha_{2}+\alpha_{3}$, we deduce:

Corollary 5 For a metric on a three manifold evolving under the Ricci flow and with normalized initial conditions, at each point and each time it holds:

$$
R+2 \phi(R) \geq \alpha_{1}, \alpha_{2}, \alpha_{3} \geq-\phi(R)
$$

It is relevant to notice that, by construction, $\lim _{y \rightarrow+\infty} \frac{\phi(y)}{y}=0$, therefore for $R \gg 1, R$ controls the whole curvature tensor.

\section{Singularities}

In this section we first explain that the scalar curvature blows up when there is a singularity. The idea of Hamilton is to take a rescaled limit of a sequence of flows when the time converges to the singular time, rescaling so that the scalar curvature becomes 1 . The main technical issue here is to control the injectivity radius of the base point of this rescaled sequence: this is what Perelman achieved with his $\kappa$-non-collapse theorem. Those limits of sequences of rescaled flows give the so called $\kappa$-solutions. They are useful to understand the singularities, and to find canonical neighborhoods of points with high scalar curvature.

\subsection{Scalar curvature}

Hamilton showed that in the three dimensional case, the scalar curvature $R$ controls the singularities:

Theorem 18 Let $M$ be a 3-manifold. Assume that the Ricci flow is defined in time $[0, T)$ and $T>0$ is maximal, then $\sup _{M} R \rightarrow \infty$ as $t \rightarrow T$.

Notice that $M$ is not assumed to be complete, thus this allows to localize singularities at points where $R \rightarrow \infty$.

The proof of this theorem has two steps. The first one applies in any dimension: the curvature tensor controls the evolution of the metric; the second one is specific to dimension three, $R$ controls the eigenvalues of the curvature $\alpha_{i}$, so that any $\alpha_{i} \rightarrow \infty$ if and only if $R \rightarrow \infty$.
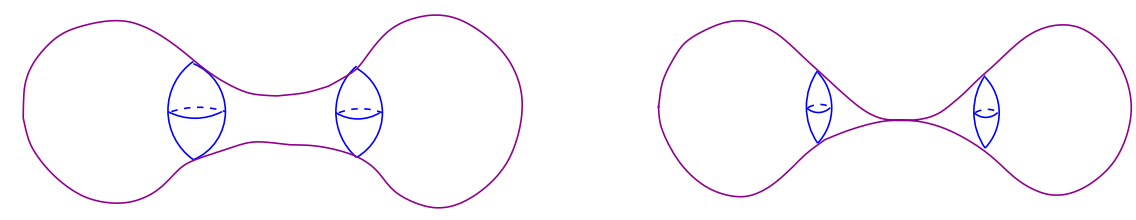

Figure 7: $S^{3}$ with a neck develops a singularity, and the curvature goes to infinity there

\subsection{Parabolic rescaling and blow ups}

To analyze singularities we need to rescale.

Definition 10 A parabolic rescaling of the metrics $g(t)$ evolving by $\frac{\partial}{\partial t} g(t)=-2$ Ric is $\lambda g\left(\frac{t}{\lambda}\right)$ for some $\lambda>0$. 

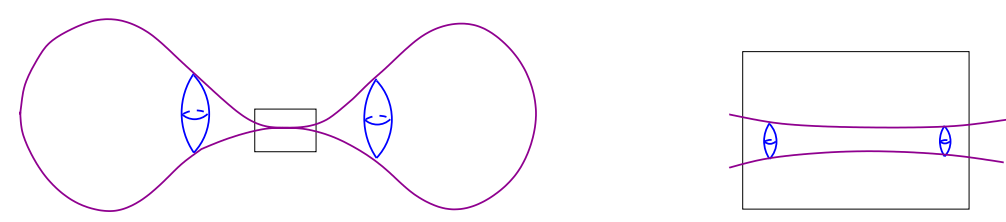

Figure 8: Rescaled limits of singularities can be though as zooms

The Ricci tensor is invariant by homoteties, thus the rescaled metric still satisfies the Ricciflow equation. The distance is multiplied by $\sqrt{\lambda}$ and the time by $\lambda$, this is why this rescaling is called parabolic.

Assume we have a singularity developing at time $T$ under the Ricci flow $(M, g)$. We take a sequence $x_{i} \in M$ and $t_{i} \rightarrow T$ so that $R\left(x_{i}\right)=\max _{M} R$ at time $t_{i}$, and we parabolic rescale to have $R\left(x_{i}\right)=1$. We also move the time by a translation, so that the initial $t_{i}$ becomes zero. In order to analyze the singularity, the idea is to look at the limit of pointed Ricci flows (with base point $x_{i}$ at time 0 , after the time translation).

There is a compactness theorem for pointed Ricci flows [19], provided we have a positive lower bound on the injectivity radius of the base point. If we had this lower bound, then there would be a convergent subsequence to a flow, and Hamilton work would yield that the limit has the following properties:

- It is an ancient solution, i.e. defined on time $(-\infty, 0]$.

- The metric is complete.

- The sectional curvature is non-negative, because we rescale by $R$ and we apply Hamilton-Ivey pinching (Corollary 5 .

- $R \geq 0$, because $R_{\min }$ is nondecreasing.

\section{3 $\kappa$-noncollapse}

Perelman uses a technique called $\kappa$-noncollapse to control the injectivity radius:

Theorem 19 ( $\kappa$-noncollapse [41]) Given a manifold $\left(M, g_{0}\right)$ there exist $\kappa>0$ and $r_{0}>0$ with the following property. Assume that $g_{t}$ evolves by the Ricci flow. Then for every $x \in M^{3}$, every $0<r<r_{0}$ and every $t \in[1, T)$, if $|R| \leq r^{-2}$ on $B(x, r) \subset\left(M, g_{t}\right)$, then

$$
\frac{\operatorname{vol}(B(x, r))}{r^{3}} \geq \kappa
$$

When the conclusion of the theorem holds, we say that the solution is $\kappa$-non collapsed at scale $\leq r_{0}$. We make the following remarks:

- This inequality gives a lower bound on the injectivity radius after normalizing to $|R| \leq 1$, by Cheeger's propeller's lemma [7].

- Notice that $\operatorname{vol}(B(x, r)) / r^{3}$ is scale invariant. By the previous remark, the scale such that $|R|=1$ is the right one to take limits.

- Notice also that this $\kappa>0$ and the scale $r_{0}$ depend on the initial conditions $\left(M, g_{0}\right)$, and that it applies to times $t \geq 1$ (i.e. once the Ricci flow has evolved for some time).

The proof is too long to be given here. It develops new functionals that are monotonic for the Ricci flow, and can be found in the original paper [41], the notes [24], [33] and [5], or the book [36]. 
If the dimension is different from 3 , then the theorem also holds true, by replacing the scalar curvature by the whole curvature tensor.

In the study of singularities, the rescaled limits satisfy the conclusion of the theorem (with $r_{0}=\infty$, because of the blow up). They are called $\kappa$-solutions:

Definition 11 A $\kappa$-solution is a solution to the Ricci flow which is:

- ancient (defined on some time interval containing $(-\infty, 0])$;

- non flat and has non-negative sectional curvature;

- metrically complete;

- $\kappa$-non-collapsed at all scales (i.e. satisfying the conclusion of Theorem 19 with $r_{0}=\infty$ ).

The cigar soliton is $\kappa$-collapsed (when we normalize at some point $R=1$, the injectivity radius is arbitrarily small). The cylinder is a $\kappa$-solution.

\subsection{Canonical neighborhoods}

Of course $\kappa$-solutions play a central role as local model for singularities, i.e. as the limit after rescaling.

Namely, points where $R$ is large, are approximate by $\kappa$ solutions. Then one has to find structure results for $\kappa$-solutions. Even if $\kappa$-solutions are more complicated than cylinders, the strategy is to find regions in the $\kappa$-solutions which are close to cylinders, in order to be able to cut along connected sums. In fact, Perelman uses $\kappa$-solutions to prove [42]:

Theorem 20 Given a manifold $\left(M, g_{0}\right)$ there exists $r>0$ and $\varepsilon>0$ with the following property:

If $t \geq 1$ and $x \in\left(M, g_{t}\right)$ satisfies $R(x) \geq \frac{1}{r^{2}}$, then $x$ lies in a canonical neighborhood.

Definition 12 A canonical neighborhood is $\varepsilon$-close to one of the following:

a. A strong $\varepsilon-n e c k$.

b. An open ball with a cylindrical end (called a cap, cf. Fig. 9).

c. A manifold diffeomorphic to $S^{3}$ or $\mathbb{R} \mathbf{P}^{3}$.

d. A manifold with positive sectional constant curvature.

Definition $13 A$ strong $\varepsilon$-neck is a neighborhood $U \subset M \times\left[0, t_{0}\right]$ of $\left(x_{0}, t_{0}\right)$

$$
U=\left\{(x, t) \in M \times\left[0, t_{0}\right] \mid d_{t_{0}}\left(x, x_{0}\right) \leq r / \varepsilon, t_{0}-r^{2} \leq t \leq r\right\}
$$

where $d_{t_{0}}$ denotes the distance at time $t_{0}$, such that after parabolic rescaling by $1 / r$, it is $1 / \varepsilon$-close to the evolving cylinder $S^{2} \times\left[-\frac{1}{\varepsilon}, \frac{1}{\varepsilon}\right]$ in a time interval of length one.

The normalization is chosen so that the scalar curvature of the cylinder at the final time is one, so after the homotety $R\left(x_{0}, t_{0}\right)=\frac{1}{r^{2}}$.

The idea now will be to do surgery along strong necks (i.e. to decompose along connected sums of these spheres). 


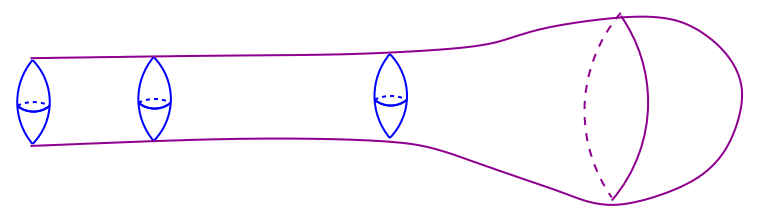

Figure 9: A cap

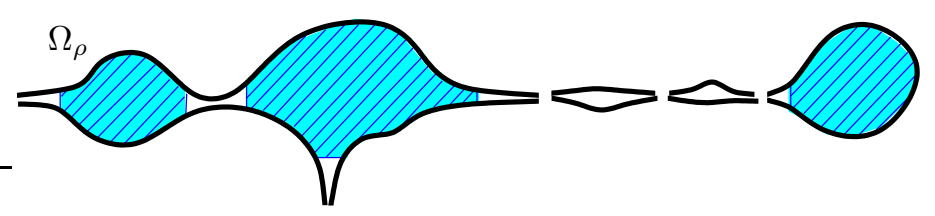

Figure 10: By 21] the complement of $\Omega_{\rho}$ has simple topology

\subsection{Gluing canonical neighborhoods}

Let $\left(M, g_{t}\right)$ denote a family of metrics evolving by the Ricci flow in a time interval $[0, T)$, with $T$ maximal. Let $R(x, t)$ denote the scalar curvature at $x$ at time $t$. Having in mind Theorem 20 we define, for some $t \approx T$

$$
\Omega_{\rho, t}=\left\{x \in M \mid R(x, t) \leq \frac{1}{\rho^{2}}\right\} .
$$

and $\Omega_{t}=\bigcup_{\rho>0} \Omega_{\rho, t}$. By the bound of the curvature and the $\kappa$-non collapse, there is a limit metric on $\Omega_{t}$ but the set $M \backslash \Omega_{t}$ can be very wild.

If $t \approx T$, every point in $M \backslash \Omega_{t}$ has a canonical neighborhood as in Theorem 20] By using the metric properties of such a neighborhood one can prove:

Theorem 21 There exists a $\delta_{0}>0$ such that if $0<\delta<\delta_{0}$ and $\rho \leq r \delta$, then $M \backslash \Omega_{\rho, t}$ is diffeomorphic to a finite union of cylinders, hats or manifolds with positive curvature.

In addition, there exist parameters $0<h \leq r \delta$ and $D>0$ such that if $x, y, z \in M, g(t)$ satisfy $R(x) \leq 1 / r^{2}, R(y) \geq D / h^{2}$ and $z$ lies in the minimizing segment between $x$ and $y$ and $R(z)=1 / h^{2}$, then $z$ is the center of a strong neck.

This theorem tells that points of high curvature lie in canonical regions, and that the transition between points of high curvature and other points is made along centers of strong necks.

After cutting along the spheres in the centers of strong necks, the surgery consists in gluing balls with some particular metrics. Those balls are obtained by gluing a long cylinder with the unit ball:

$$
S^{2} \times[0,10] \cup B^{3}
$$

where $S^{2} \times\{10\}$ is identified to $\partial B^{3}$ (Figure 11). The metric around the gluing sphere $S^{2} \times\{10\}=\partial B^{3}$ must be regularized without changing too much the bounds on the curvature. A homotety of factor $h$ is required in order to have the same diameter as the spheres in $\partial \Omega_{h}$.

We do not assume that the manifold is connected, new components may appear after surgery. Some of the new components can have positive curvature (and satisfy geometrization conjecture, by Hamilton's Theorem, Thm. 16.

Notice that Theorem 21] allows to cut along spheres in such a way that the topology of the complement of $\Omega_{\rho}$ is simple enough. In addition the metric is well understood on the boundary of $\Omega_{\rho}$.

The idea is to restart the flow and apply the process again. In order to do that, we must be careful with the constants and we will define a flow with cutoff. 


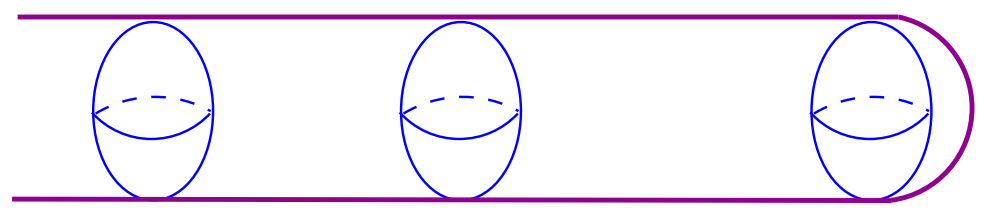

Figure 11: The piece we add after cutoff
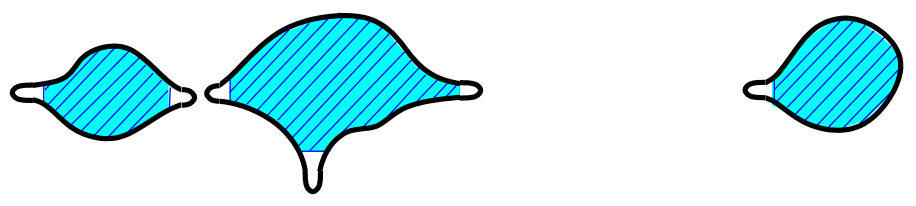

Figure 12: The manifold after cutoff (cf. Fig. 10,

\section{Flow with cutoff}

The definition here follows [1], where we consider some cutoff times, which are not singular, but just before the singular times, so that we already have quite high curvature. This is a technical modification of Perelman's [42] that does not change the main ideas of the proof, but allows to simplify some technical issues.

Definition 14 A flow with cutoff is a family of Riemannian three manifolds $\left(M_{t}, g(t)\right)$ parametrized by $t \in[0, T), T \in \mathbb{R}_{+} \cup\{\infty\}$, such that there exists a discrete set in $\mathbb{R}$ of cutoff times $0<t_{1}<\cdots<t_{n}<$ $\cdots<T$ satisfying:

a. For $t \in\left[t_{i-1}, t_{i}\right),(M(t), g(t))$ is a solution to the Ricci flow equation. In particular the topology of $M(t)$ does not change along $\left[t_{i-1}, t_{i}\right)$.

b. The solution in the interval $\left[t_{i-1}, t_{i}\right)$ can be extended to time $t_{i}$, and we denote it by $\left(M_{-}\left(t_{i}\right), g_{-}\left(t_{i}\right)\right)$.

c. $\left(M\left(t_{i}\right), g\left(t_{i}\right)\right)$ is the result of cutoff of $\left(M_{-}\left(t_{i}\right), g_{-}\left(t_{i}\right)\right)$ as in Theorem 21 for some parameters $r, \delta$, $h>0$.

We say that a manifold has normalized initial conditions if at time $0 R \geq-1$ and satisfies the HamiltonIvey pinching condition. The folowing result tells that the Ricci flow with cutoff exists, and it can be prolonged for infinite time except if for some time the whole manifold satisfies the conclusion of Theorem 21

Theorem 22 There exist positive decreasing functions $r, \delta, h:[0,+\infty) \rightarrow(0, \infty)$ such that for any Riemannian irreducible three manifold with normalized metric $(M, g(0))$, one of the following holds.

a. Either there exists $t_{0}>0$ such that a flow with cutoff $(M, g(t))$ is defined on $\left[0, t_{0}\right)$ and $\Omega_{\rho\left(t_{0}\right), t_{0}}=M$.

b. Or a flow with cutoff $(M, g(t))$ and parameters $r, \delta$, $h$ is defined on $[0, \infty)$.

Some remarks about this theorem are in order. Firstly, there can be infinitely many cutoff times, but they do not accumulate, by a volume argument. Namely, the time derivative of volume follows the rule of Lemma2

$$
\frac{d}{d t} \operatorname{Vol}(M, g(t))=-\int_{M} R
$$


Since the minimum on $M$ of the scalar curvature $R$ is non-decreasing, the growth of $\operatorname{Vol}(M, g(t))$ is controlled. On the other hand, one can estimate the volume lost at each cutoff, and the conclusion is that for a sufficiently large amount of cutoff times in a bounded interval, the volume would become zero.

Secondly, the constants $\delta>0, r>0$ and $h>0$ change at every cutoff time. One has to show that they do not converge to zero in finite time. In addition the choice of parameters $\delta>0, r>0$ and $h>0$ can modify the flow, hence there is no uniqueness. The proof is by contradiction, and the constants are not constructible.

Thirdly, the cutoff process is metric, but the connected sums can be topologically trivial (as in the example of Figure 13.

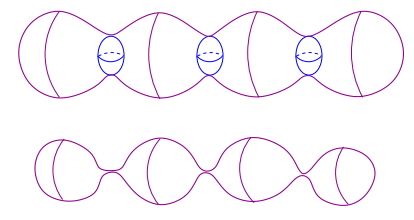

Figure 13: Topologically trivial connected sums produced by the flow.

It is unclear if the number of cutoff times is finite or infinite. No example with infinitely many times is know yet.

Definition 15 We declare the flow extinct when at some time $t_{0}$, we have $\Omega_{\rho\left(t_{0}\right), t_{0}}=M$, i.e. as in Theorem 22]

When the flow becomes extinct, by Theorem 21 the manifold is a connected sum of pieces of constant curvature 1 and $S^{2} \times S^{1}$. In particular it is the connected sum of Seifert manifolds and satisfies the geometrization conjecture.

\section{Elliptization}

Perelman wrote a third paper [43], where he proves that for elliptization (in particular for proving Poincaré conjecture) the long time behaviour is not required.

Theorem 23 If the initial manifold has finite or trivial fundamental group, then the flow with cutoff becomes extinct.

We recall that the flow becomes extinct when, at some finite time, $\Omega_{\rho}=\emptyset$.

Even if Perelman gave a proof with loops, we will follow the approach of Colding and Minicozzi [10] [1], with sweepouts, families of maps from the sphere $S^{2}$ to the manifold $M^{3}$. Both proofs use that, when $\pi_{1}\left(M^{3}\right)$ is finite, then $\pi_{3}\left(M^{3}\right) \neq 0$.

IDEA OF PROOF. We consider the space of continuous maps $S^{2} \rightarrow M^{3}$ whose differential is square integrable:

$$
\Theta:=L_{1}^{2}\left(S^{2}, M^{3}\right) \cap C^{0} .
$$

Notice than $M^{3}$ can be embedded into $\Theta$ by means of the constant map: to each $x \in M^{3}$ it corresponds the constant map from $S^{2}$ to $x$. Therefore we can consider the relative fundamental group:

$$
\pi_{1}\left(\Theta, M^{3}\right)
$$

which is the set of homotopy classes of maps from the interval $[0,1]$ to $\Theta$, such that the endpoints are in $M^{3}$ (i.e. the endpoints are constant maps). 
Since $\pi_{1}\left(M^{3}\right)$ is either trivial or finite, Hurewicz theorem applied to its universal covering tells us that $\pi_{3}\left(M^{3}\right) \cong \mathbb{Z}$. This implies that $\pi_{1}\left(\Theta, M^{3}\right) \neq 0$, because if we have a map $f: S^{3} \rightarrow M^{3}$ which is not homotopically trivial, and we assume that $f$ is of class $\mathcal{C}^{2}$, then we consider the height function on $S^{3} \subset \mathbb{R}^{4}$, which is a Morse function, and its level sets are a family of spheres $S^{2}$, except for both critical points, the north and the south pole, where the level sets are points, Fig. 14 This gives a path in $\Theta$, with parameter the height function, with endpoints in $M^{3} \subset \Theta$. Clearly, it is a nontrivial element in $\pi_{1}\left(\Theta, M^{3}\right)$, because it comes from a nontrivial element in $\pi_{3}\left(M^{3}\right)$.

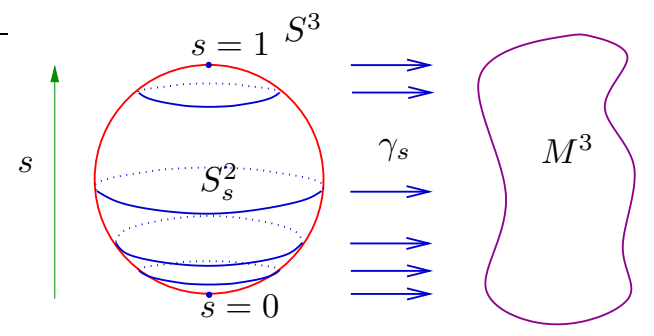

Figure 14: Constructing element in $\pi_{1}\left(\Theta, M^{3}\right)$ from an element in $\pi_{3}\left(M^{3}\right)$.

Let $\theta \in \pi_{1}\left(\Theta, M^{3}\right)$ denote a nontrivial element. Colding and Minicozzi define the width:

$$
W(g, \theta)=\min _{\gamma \in \theta} \max _{s \in[0,1]} E\left(\gamma_{s}\right)>0, \quad \text { where } E\left(\gamma_{s}\right)=\frac{1}{2} \int_{S^{2}}\left\|d \gamma_{s}\right\|^{2} d \mu_{S^{2}} .
$$

Equivalently, given a path in $\Omega$ with endpoints in $M^{3}$, they consider the maximun of the energy among all values of the parameter $s \in[0,1]$. Then they take the minimum among all paths that represent the given homotopy class $\theta \neq 0$.

By a theorem of Jost [22], $W(g, \theta)>0$ for $\theta \in \pi_{1}(\Theta, \Omega)$ non trivial.

When $g(t)$ evolves by the Ricci flow with cutoff, Colding and Minicozzi prove [10] 11]:

$$
\frac{d}{d t} W(g(t), \theta) \leq-4 \pi+\frac{3}{4(t+C)} W(g(t), \theta)
$$

that has a meaning even when it is not differentiable. This inequality implies that for some finite time $t_{0}$, $W\left(g\left(t_{0}\right), \theta\right)=0$. Since this contradicts Jost's result $W(g, \theta)>0$, the only possibility is that the flow is not defined at time $t_{0}$, i.e. it has become extinct.

\section{Long time behaviour}

We assume thus that the flow can be continued during infinite time. We denote by $M_{t}$ the manifold at time $t$, that may have changed topologically because of cutoff.

\subsection{Thin thick decomposition}

We want to decompose the manifold as the union of hyperbolic and Seifert fibered pieces. According to Cheeger and Gromov's theory (Theorem 9, the Seifert pieces should correspond to pieces with small injectivity radius with respect to curvature (thin part). In addition, by Margulis lemma (cf. [51]), hyperbolic manifolds have injectivity radius uniformly minorated (thick part). We want to find a decomposition into thin and thick parts, using scale invariant notions.

For every time $t \gg 1$ we define

$$
\eta(x, t)=\sup \left\{r>0 \mid \text { the ball } B(x, r) \subset M_{t} \text { has curvature } \geq-\frac{1}{r^{2}}\right\} .
$$


We fix a constant $\omega>0$. We will say that $x \in M_{t}^{-}(\omega)$ if

$$
\operatorname{vol}\left(B(x, \eta(x, t)) \leq \omega \eta(x, t)^{3} .\right.
$$

Definition 16 The $\omega$-thin part is $M_{t}^{-}(\omega)$, and the $\omega$-thick $M_{t}^{+}(\omega)=M \backslash M_{t}^{-}(\omega)$.

Perelman uses the regularity properties of the flow to prove:

Theorem 24 For every $\omega>0$, the $\omega$-thick part becomes stable (not affected by cuttoff for large times) and of asymptotically constant negative curvature.

Thus the components of the thick part, when normalized by $\sqrt{t}$, converge to manifolds of constant negative curvature, moreover, by Corollary 4 that applies to the flow with cutoff, they have finite volume. Hence their boundary components are tori, by Margulis lemma. One may ask whether they are tori of the JSJ decomposition or not. This is equivalent to ask whether they are incompressible or not (i.e. $\pi_{1}$-injective).

Theorem 25 The boundary tori of the thick part are incompressible.

For this theorem Perelman adapts an argument of Hamilton [20] to the case with cutoff. By contradiction, assume that one of the torus is compressible. According to Dehn lemma, there exists a compressing disc, i.e. a disc in the manifold whose boundary is and essential curve of the torus. A theorem of Meeks and Yau [28] allows to choose a minimal smooth embedded disk. Hamilton shows that the area of the disc decreases at a rate bounded away from zero, which is impossible for a flow with cutoff defined for infinite time.

Remark 3 This concludes the proof of geometrization when the thick part is non empty. If the thick part has no boundary, then the flow converges to a hyperbolic metric, after renormalizing. If it has boundary, then the manifold contains incompressible tori and therefore it is sufficiently large, and one can apply Thurston's geometrization (Theorem [5).

\subsection{Collapses and geometrization of aspherical manifolds}

The thin part of the manifold is expected to be a graph manifold, but Cheeger-Gromov theory cannot be applied here because there is no upper bound for the curvature.

Here we have to quote a paper of Shioya and Yamaguchi [49], in which they show that the thin part is a graph manifold. Their proof relies on Alexandrov spaces. Those are metric spaces satisfying the same properties and inequalities for distances as in a manifold with lower curvature bound. Unfortunately, their proof uses unpublished results of Perelman himself, as well as generalizations of the results of Shioya and Yamaguchi, not explicitly written up. We propose in [1] a different approach, that we explain here.

Theorem 26 Let $\left(M_{n}^{3}, g_{n}\right)$ be a sequence of aspherical Riemannian three manifolds satisfying.

(a). Volume collapse. There exists a sequence $\varepsilon_{n} \rightarrow 0$ such that for each $x \in M_{n}$, there is a radius $0<\rho_{n}(x)$ satisfying

$$
\sec \geq-\frac{1}{\rho_{n}(x)^{2}} \text { on } B\left(x, \rho_{n}(x)\right) \quad \text { and } \quad \operatorname{Vol}\left(B\left(x, \rho_{n}(x)\right)\right) \leq \varepsilon \rho_{n}(x)^{3} .
$$

(b). Local curvature bounds. For every $\delta>0 \exists \bar{r}=\bar{r}(\delta)>0$ such that: If $0<r<\bar{r}(\delta), \frac{\operatorname{Vol}(B(x, r))}{r^{3}}>\delta$ and $\sec \geq-\frac{1}{r^{2}}$ on $B(x, r)$, then

$$
|R m|<K_{0} r^{-2} \quad \text { and } \quad|\nabla R m|<K_{1} r^{-3} .
$$


Then for $n$ sufficiently large, $M_{n}$ is a graph manifold.

This is a modified version of Theorem 7.4 of Perelman [42].

By Theorem 23 we may assume that the manifold is aspherical. As we mentioned in Remark 3 we may also assume that the thick part is empty, and hypothesis (a) says that the manifold is collapsing everywhere. Hypothesis thm:collapse:b is a regularity property of the flow, justified in 7.3 of Perelman [42], cf. [24].

Notice that hypothesis (a) and (b) are not contradictory, as they deal with different scales. Namely, since $\lim _{r \rightarrow 0} \frac{\operatorname{Vol}(B(x, r)}{r^{3}}=\frac{4 \pi}{3}$, (b) applies to a much smaller scale than (a)

The proof of Theorem 26 in [1] has three main steps. Firstly there is a local structure theorem: by a blow up argument, every point has a neighborhood that looks like a manifold of non-negative curvature. If the manifold is compact by Hamiton's theorem we are done (Remark 2). Otherwise some of the metric properties are controlled, and its topology is rather simple, by Cheeger-Gromoll soul theorem: $\mathbb{R}^{3}, T^{2} \times \mathbb{R}$, $S^{2} \times \mathbb{R}, S^{1} \times \mathbb{R}^{2}$ or $K^{2} \tilde{\times} \mathbb{R}$, the orientable line bundle over the Klein bottle. Using metric properties of the soul theorem, one can choose the local neighborhoods diffeomorphic to the whole manifold.

The second step is to shrink this covering to have dimension two (every point belongs to at most three open sets). To do it, we use a nice metric argument of Gromov [14], where the volume notion of collapse (hypothesis (a) in the theorem) is used to shrink coverings, also as in [4].

Lemma 3 Suppose that an aspherical closed n-manifold $N^{n}$ admits an open covering $\left\{U_{i}\right\}_{i \in I}$ such that for every $i \in I, \pi_{1}\left(U_{i}\right) \rightarrow \pi_{1}\left(M^{n}\right)$ is trivial. Then the dimension of the covering $\left\{U_{i}\right\}_{i \in I}$ is at least $n$ (i.e. at least one point belongs to $n+1$ open sets).

This lemma uses asphericity, for instace it is trivially not true for a sphere. The proof uses a Čech-de Rham complex to compute

$$
H^{n}\left(N^{n}, \mathbb{R}\right) \cong H^{n}\left(\pi_{1}\left(N^{n}\right), \mathbb{R}\right) .
$$

If the dimension of the covering was less than or equal to $n$, then $H^{n}\left(N^{n}, \mathbb{R}\right)=0$, and therefore it has to be at least $n+1$, cf. [27]. Notice that the orientability of $N^{n}$ can be assumed after taking the orientable covering.

Since our covering has dimension two for at least one of the open sets $U_{0}$ it holds that $\pi_{1}\left(U_{0}\right) \rightarrow$ $\pi_{1}\left(M^{3}\right)$ is nontrivial, by Lemmaß In particular $U_{0} \cong T^{2} \times \mathbb{R}, S^{1} \times \mathbb{R}^{2}$ or $K^{2} \tilde{\times} \mathbb{R}$. By standard threedimensional topology, the manifold $M^{3} \backslash U_{0}$ is irreducible ( $U_{0}$ is not contained in a ball), it is sufficiently large and has boundary consisting of tori.

We apply Thurston's geometrization, and therefore $M \backslash U_{0}$ has a JSJ decomposition with Seifert and hyperbolic pieces. We claim that there are not hyperbolic pieces. This is proved by means of the simplicial volume (also called Gromov norm). By Gromov-Soma [14 [50], the simplicial volume equals a constant multiple of the addition of volumes of hyperbolic pieces. Since we have this covering of dimension two by open sets whose fundamental group is trivial, Gromov's vanishing theorem implies that the simplicial volume of $M^{3} \backslash U_{0}$ vanishes. Thus $M^{3} \backslash U_{0}$ is a graph manifold and so is $M^{3}$. By Waldhausen $M^{3}$ is a graph manifold: either Seifert fibered or with only Seifert pieces in the JSJ decomposition.

Acknowledgement. My research is partially supported by FEDER/MEC through grant MTM200604353.

I am indebted to the Fundación Areces and the Real Academia de Ciencias Exactas, Físicas y Naturales de Madrid, for their invitation to the symposiun Mathematics for the XXIst Century. I also thank many people who helped me to understand the flow and Perelman's work: L. Bessières, G. Besson, E. Cabezas, C. Curràs, J. Dinkelbach, B. Leeb, S. Maillot, E. Miranda, I. Mundet, M. Nicolau, V. Miquel and $\mathrm{H}$. Weiss.

\section{References}

[1] Bessières, L., Besson, G., Boileau, M., Maillot, S. M. and Porti, J., (2007). Weak collapsing and geometrisation of aspherical 3-manifolds, (preprint). 
[2] Bianchi, L., (1897). Sugli spazi a tre dimensioni che ammettono un gruppo continuo di movimenti, Mem. Soc. Ital. Scienze, 11, 3, 267-352.

[3] Bing, R. H., (1954). Locally tame sets are tame, Ann. of Math. (2), 59, 145-158.

[4] Boileau, M., Leeb, B. and Porti, J., (2005). Geometrization of 3-dimensional orbifolds, Ann. of Math. (2), 162, 1, 195-290.

[5] Cao, H.-D. and Zhu, X.-P., (2006). A complete proof of the Poincaré and geometrization conjectures-application of the Hamilton-Perelman theory of the Ricci flow, Asian J. Math., 10, 2, 165-492.

[6] Casson, A. and Jungreis, D., (1994). Convergence groups and Seifert fibered 3-manifolds, Invent. Math., 118, 3 , 441-456.

[7] Cheeger, J. and Ebin, D. G., (1975). Comparison theorems in Riemannian geometry, North-Holland Publishing Co., Amsterdam. North-Holland Mathematical Library, 9.

[8] Cheeger, J. and Gromov, M., (1986). Collapsing Riemannian manifolds while keeping their curvature bounded. I, J. Differential Geom., 23, 3, 309-346.

[9] Cheeger, J. and Gromov, M., (1990). Collapsing Riemannian manifolds while keeping their curvature bounded. II, J. Differential Geom., 32, 1, 269-298.

[10] Colding, T. H. and Minicozzi, II, W. P., (2005). Estimates for the extinction time for the Ricci flow on certain 3-manifolds and a question of Perelman, J. Amer. Math. Soc., 18, 3, 561-569, (electronic).

[11] Colding, T. H. and Minicozzi, II, W. P., (2007). Width and finite extinction time of Ricci flow. (preprint).

[12] DeTurck, D. M., (1983). Deforming metrics in the direction of their Ricci tensors, J. Differential Geom., 18, 1, $157-162$.

[13] Gabai, D., (1992). Convergence groups are Fuchsian groups, Ann. of Math. (2), 136, 3, 447-510.

[14] Gromov, M., (1982). Volume and bounded cohomology, Inst. Hautes Études Sci. Publ. Math., 56, 5-99.

[15] Gromov, M. and Lawson, H. B., Jr., (1980). The classification of simply connected manifolds of positive scalar curvature, Ann. of Math. (2), 111, 3, 423-434.

[16] Haken, W., (1962). Über das Homöomorphieproblem der 3-Mannigfaltigkeiten. I, Math. Z., 80, 89-120.

[17] Hamilton, R. S, (1982). Three-manifolds with positive Ricci curvature, J. Differential Geom., 17, 2, $255-306$.

[18] Hamilton, R. S., (1986). Four-manifolds with positive curvature operator, J. Differential Geom., 24, 2, 153-179.

[19] Hamilton, R. S., (1995). The formation of singularities in the Ricci flow, in Surveys in differential geometry, Vol. II (Cambridge, MA, 1993), Int. Press, Cambridge, MA, 7-136.

[20] Hamilton, R. S., (1999). Non-singular solutions of the Ricci flow on three-manifolds, Comm. Anal. Geom., 7, 4, $695-729$.

[21] Ivey, T., (1993). Ricci solitons on compact three-manifolds, Differential Geom. Appl., 3, 4, 301-307.

[22] Jost, J., (1991). Two-dimensional geometric variational problems, Pure and Applied Mathematics (New York), John Wiley \& Sons Ltd., Chichester., A Wiley-Interscience Publication.

[23] Kapovich, M., (2001). Hyperbolic manifolds and discrete groups, Progress in Mathematics, 183, Birkhäuser Boston Inc., Boston, MA.

[24] Kleiner, B. and Lott, J. Notes on Perelman's papers, Preprint, math. DG/0605667.

[25] Kneser, H., (1929). Geschlossene Flächen in dreidimensionalen Mannigfaltigkeiten., Jahresbericht D. M. V., 38, $248-260$. 
[26] McMullen, C., (1990). Iteration on Teichmüller space, Invent. Math., 99, 2, 425-454.

[27] McMullen, C. T., (2005). Minkowski's conjecture, well-rounded lattices and topological dimension, J. Amer. Math. Soc., 18, 3, 711-734, (electronic).

[28] Meeks III, W. H. and Yau, S. T., (1981). The equivariant Dehn's lemma and loop theorem, Comment. Math. Helv., 56, 2, 225-239.

[29] Mess, G., The Seifert conjecture and groups which are coarse quasiisometric to planes, (preprint).

[30] Milnor, J., (2003). Towards the Poincaré conjecture and the classification of 3-manifolds, Notices Amer. Math. Soc., 50, 10, 1226-1233.

[31] Moise, E. E., (1952). Affine structures in 3-manifolds. V. The triangulation theorem and Hauptvermutung, Ann. of Math, (2), 56, 96-114.

[32] Montesinos-Amilibia, J. M., (1984). Punts de vista sobre el problema de Poincaré, in The development of mathematics in the nineteenth century, Arx. Sec. Cièn., LXXV, Inst. Estudis Cat., Barcelona, 23-39.

[33] Morgan, J. and Tian, G., Ricci Flow and the Poincare Conjecture, math. DG/0607607, (preprint).

[34] Morgan, J. W. and Shalen, P. B., (1984). Valuations, trees, and degenerations of hyperbolic structures. I, Ann. of Math. (2), 120, 3, 401-476.

[35] Mostow, G. D., (1968). Quasi-conformal mappings in $n$-space and the rigidity of hyperbolic space forms, Inst. Hautes Études Sci. Publ. Math., 34, 53-104.

[36] Müller, R., (2006). Differential Harnack inequalities and the Ricci flow, EMS Series of Lectures in Mathematics, European Mathematical Society (EMS), Zürich.

[37] Munkres, J., (1960). Obstructions to the smoothing of piecewise-differentiable homeomorphisms, Ann. of Math. (2), 72, 521-554.

[38] Myers, R., (1982). Simple knots in compact, orientable 3-manifolds, Trans. Amer. Math. Soc., 273, 1, 75-91.

[39] Otal, J.-P., (1996). Le théorème d'hyperbolisation pour les variétés fibrées de dimension 3, Astérisque, , 235 , $\mathrm{x}+159$.

[40] Otal, J.-P., (1998). Thurston's hyperbolization of Haken manifolds, in Surveys in differential geometry, III (Cambridge, MA, 1996), Int. Press, Boston, MA, 77-194.

[41] Perelman, G., The entropy formula for the Ricci flow and its geometric applications, math. DG/0211159, (preprint).

[42] Perelman, G., Ricci flow with surgery on three-manifolds, math. DG/0303109, (preprint).

[43] Perelman, G., Finite extinction time for the solutions to the Ricci flow on certain three-manifolds, math. DG/0307245, (preprint).

[44] Poincaré, H., (1904). Cinquième complément à l'analysis situs, Palermo Rend., 18, 45-110.

[45] Riley, R., (1975). A quadratic parabolic group, Math. Proc. Cambridge Philos. Soc., 77, 281-288.

[46] Schoen, R. and Yau, S. T., (1979). Existence of incompressible minimal surfaces and the topology of threedimensional manifolds with nonnegative scalar curvature, Ann. of Math. (2), 110, 1, 127-142.

[47] Scott, P., (1983). The geometries of 3-manifolds, Bull. London Math. Soc., 15, 5, 401-487.

[48] Seifert, H., (1933). Topologie dreidimensionaler gefaserter Räume., Acta Math., 60, 147-238.

[49] Shioya, T. and Yamaguchi, T., (2005). Volume collapsed three-manifolds with a lower curvature bound, Math. Ann., 333, 1, 131-155. 
[50] Soma, T., (1981). The Gromov invariant of links, Invent. Math., 64, 3, 445-454.

[51] Thurston, W., (1979). The geometry and topology of 3-manifolds, Princeton Math. Dept., Princeton NJ.

[52] Thurston, W. P., (1982). Three-dimensional manifolds, Kleinian groups and hyperbolic geometry, Bull. Amer. Math. Soc. (N.S.), 6, 3, 357-381.

[53] Thurston, W. P., (1986). Hyperbolic structures on 3-manifolds. I. Deformation of acylindrical manifolds, Ann. of Math. (2), 124, 2, 203-246.

[54] Thurston, W. P., (1986), Hyperbolic structures on 3-manifolds. II: Surface groups and 3-manifolds which fiber over the circle. (preprint).

[55] Thurston, W. P., (1986), Hyperbolic structures on 3-manifolds. III: Deformations of 3-manifolds with incompressible boundary. (preprint).

[56] Thurston, W. P., (1988). On the geometry and dynamics of diffeomorphisms of surfaces, Bull. Amer. Math. Soc. (N.S.), 19, 2, 417-431.

[57] Tukia, P., (1988). Homeomorphic conjugates of Fuchsian groups, J. Reine Angew. Math., 391, 1-54.

[58] Waldhausen, F., (1967). Eine Klasse von 3-dimensionalen Mannigfaltigkeiten. I, II, Invent. Math., 3, (1967), 308-333; ibid., 4, 87-117.

[59] Waldhausen, F., (1968). On irreducible 3-manifolds which are sufficiently large, Ann. of Math. (2), 87, 56-88.

[60] Whitehead, J., (1935). A certain open manifold whose group is unity., Q. J. Math., Oxf. Ser., 6, 268-279.

\section{Joan Porti}

Departament de Matemàtiques, Universitat Autònoma de Barcelona 08193 Cerdanyola del Vallès, Spain portiemat.uab.cat 